\title{
Expression to predict the chromatic dispersion of a high- order mode in few-mode fibers
}

\author{
Masaharu Ohashi ${ }^{1, \text { a) }}$, Tomoya Kawasaki ${ }^{2}$, Hirokazu Kubota ${ }^{2}$, \\ Yuji Miyoshi $^{2}$, Atsushi Nakamura ${ }^{3}$, and Daisuke Iida ${ }^{3}$ \\ 1 Department of Technological Systems, Osaka Prefecture University College of \\ Technology
}

Saiwai-cho, Neyagawa, Osaka, 572-8572, Japan

${ }^{2}$ Graduate School of Engineering, Osaka Prefecture University

Gakuen-cho, Naka, Sakai, Osaka 599-8531, Japan

${ }^{3}$ Access Network Service Systems Laboratories, NTT Corporation

Hanabatake, Tsukuba, Ibaraki 305-0805, Japan

a) ohashi@eis.osakafu-u.ac.jp

\begin{abstract}
A general expression for the waveguide dispersion of a propagation mode is derived from the wave equation. Furthermore, a simple expression for the waveguide dispersion of high-order modes is derived as a function of the mode field radius by substituting an approximated function of the electrical field distribution of the mode into the general expression. The chromatic dispersions of the $\mathrm{LP}_{01}, \mathrm{LP}_{11}, \mathrm{LP}_{02}$, and $\mathrm{LP}_{21}$ modes in a fourmode fiber are estimated using the proposed expression, and their accuracies are evaluated through simulations.
\end{abstract}

Keywords: chromatic dispersion, waveguide dispersion, high-order mode, mode field radius, few-mode fiber

Classification: Optical Fiber for Communications

\section{References}

[1] D. Qian, M.-F. Huang, E. Ip, Y.-K. Huang, Y. Shao, J. Hu, and T. Wang, "High capacity/spectral effciency 101.7-Tb/s WDM transmission using PDM-128QAMOFDM over 165-km SSMF within C- and L-bands," J. Lightw. Technol., vol. 30, no. 10, pp. 1540-1548, 2012. DOI: 10.1109/JLT.2012.2189096

[2] J. Sakaguchi, W. Klaus, J.M.D. Mendinueta, B.J. Puttnam, R.S. Luis, Y. Awaji, N. Wada, T. Hayashi, T. Nakanishi, T. Watanabe, Y. Kokubun, T. Takahata, and T. Kobayashi, "Large spatial channel (36-core $\times 3$ mode) heterogeneous few-mode multi-core fiber," J. Lightw. Technol., vol. 34, no. 1, pp. 93-103, 2016. DOI: 10. 1109/JLT.2015.2481086

[3] P. Sansonetti, "Modal dispersion in single-mode fibres: simple approximation issued from mode spot size spectral behavior," Electron. Lett., vol. 18, no. 15, pp. 647-648, 1982. DOI: 10.1049/el:19820441

[4] J.D. Love and C.D. Hussey, "Variational approximations for higher-order modes of weakly-guiding fibres," Opt. Quantum Electron., vol. 16, no. 1, pp. 41-48, 1984. DOI: 10.1007/BF00619876

[5] M. Ohashi, T. Kawasaki, H. Kubota, and Y. Miyoshi, "Prediction of modal dispersion of high-order mode from wavelength dependence of mode field radius," 
Proc. 24th Optoelectronics and Communications Conference, Fukuoka, Japan, 2019. DOI: $10.23919 /$ PS.2019.8817792

[6] W.A. Gambling, H. Matsumura, and C.M. Ragdale, "Mode dispersion, material dispersion and profile dispersion in graded-index single-mode fibres," IEE J. Microw. Opt. Acoust., vol. 3, no. 6, pp. 239-246, 1979. DOI: 10.1049/ij-moa. 1979.0051

[7] A. Nakamura and D. Iida, "Mode field diameter definitions for few-mode fibers based on spot size of higher-order Gaussian mode," IEEE Photon. J., vol. 12, no. 2, 7200609, 2020. DOI: 10.1109/JPHOT.2020.2967628

[8] ITU-T Recommendation G.650.1, "Definitions and test methods for linear, deterministic attributes of single-mode fibre and cable".

[9] D. Gloge, "Dispersion in weakly guiding fiber," Appl. Opt., vol. 10, no. 11, pp. 2442-2445, 1971. DOI: 10.1364/AO.10.002442

\section{Introduction}

Internet traffic has continued to increase in recent years owing to the rapid proliferation of internet services. Recently, a large-capacity transmission of 100 Tbps has been realized using standard single-mode fibers (SSMFs) [1]. To extend the limit of the transmission capacity of SSMFs, the use of a spatial division multiplex (SDM) system using multi-core fibers and few-mode fibers (FMFs) has been extensively investigated [2]. Knowledge of the modal dispersion of high-order modes in the FMFs is of significant interest in developing SDM systems. It has been reported that the modal dispersion of the fundamental mode in a single-mode fiber can be simply predicted based on the wavelength dependence of the mode-field radius (MFR) [3]. However, the prediction of the waveguide dispersion of a high-order mode in the FMFs and its simple expression have not been adequately considered.

This paper proposes a general expression for waveguide dispersion, which can be applied to the high-order linearly polarized $\mathrm{LP}_{l m}$ mode in the FMFs. Furthermore, the general expression is simplified as a function of the MFR by approximating the electrical field as a higher-order Gaussian function [4]. A simulation was performed to verify the accuracy of the proposed simple expression of chromatic dispersion for the $\mathrm{LP}_{01}, \mathrm{LP}_{11}, \mathrm{LP}_{02}$, and $\mathrm{LP}_{21}$ modes in a four-mode fiber with a step-index profile. While part of this work has been reported [5], this paper presents more details regarding the previous investigations.

\section{Derivation of a general expression for waveguide dispersion}

The wave equation in a fiber can be expressed in cylindrical coordinates as

$$
\frac{\partial^{2} \phi_{l m}}{\partial r^{2}}+\frac{1}{r} \frac{\partial \phi_{l m}}{\partial r}+\frac{1}{r^{2}} \frac{\partial^{2} \phi_{l m}}{\partial \theta^{2}}+\left(k^{2} n^{2}(r)-\beta^{2}\right) \phi_{l m}=0 .
$$

Considering the weakly guiding approximation, the electric component $\phi_{l m}(r, \theta)$ of the linearly polarized $\mathrm{LP}_{l m}$ mode (where $l$ and $m$ denote the azimuthal and radial mode numbers, respectively) in cylindrical coordinates can be expressed as follows [6]:

$$
\phi_{l m}(r, \theta)=R_{l m}(r) \cos (l \theta) \text { or } R_{l m}(r) \sin (l \theta),
$$

where $r$ and $\theta$ denote the radial distance and azimuthal angle, respectively. 
The index profile of the fiber can be defined as

$$
n^{2}(r)= \begin{cases}n_{1}^{2}(1-2 \Delta(r)) & r \leq a \\ n_{1}^{2}\left(1-2 \Delta_{0}\right)=n_{2}^{2} & r \geq a\end{cases}
$$

where $a$ and $\Delta$ denote the core radius and relative-index difference between the core and cladding, respectively. $n_{1}$ and $n_{2}$ denote the refractive indices of the core and cladding, respectively.

Substituting Eq. (2) into Eq. (1) yields

$$
\frac{1}{r} \frac{d}{d r}\left[r \frac{d R_{l m}}{d r}\right]+\left(k^{2} n^{2}(r)-\beta^{2}-\frac{l^{2}}{r^{2}}\right) R_{l m}=0 .
$$

The normalized propagation constant $b$ and normalized frequency $v$ are defined as

$$
\begin{gathered}
b=\frac{(\beta / k)^{2}-n_{2}^{2}}{n_{1}^{2}-n_{2}^{2}}=\frac{a^{2}}{v^{2}}\left(\beta^{2}-k^{2} n_{2}^{2}\right), \\
v=a k n_{1} \sqrt{2 \Delta},
\end{gathered}
$$

where $k$ is the wave number.

Multiplying Eq. (1) by $R_{l m}$ and integrating over the cross-section $A$ of a fiber, $b$ can be expressed as

$$
b=-\frac{a^{2}}{v^{2}} \iint_{A}\left(\frac{d R_{l m}}{d r}\right)^{2} d A+\iint_{\text {core }} R_{l m}{ }^{2} d A-\iint_{\text {core }} \frac{\Delta(r)}{\Delta_{0}} R_{l m}{ }^{2} d A-\frac{a^{2}}{v^{2}} \iint_{A} \frac{l^{2}}{r^{2}} R_{l m}{ }^{2} d A .
$$

After some calculations, the normalized group delay $d(v b) / d v$ can be obtained as

$$
\frac{d(v b)}{d v}=\iint_{\text {core }} R_{l m}^{2} d A+\frac{a^{2}}{v^{2}} \iint_{A}\left(\frac{d R_{l m}}{d r}\right)^{2} d A-\iint_{\text {core }} \frac{\Delta(r)}{\Delta_{0}} R_{l m}{ }^{2} d A+\frac{a^{2}}{v^{2}} \iint_{A} \frac{l^{2}}{r^{2}} R_{l m}{ }^{2} d A .
$$

Differentiating Eq. (8) with respect to $v$ yields

$$
\begin{aligned}
\frac{d^{2}(v b)}{d v^{2}}= & \iint_{\text {core }}\left(1-\frac{\Delta(r)}{\Delta_{0}}\right) 2 R_{l m} \frac{d R_{l m}}{d v} d A-\frac{2 a^{2}}{v^{3}} \iint_{A} \dot{R}_{l m}^{2} d A+\frac{a^{2}}{v^{2}} \iint_{A} 2 \dot{R}_{l m} \frac{d \dot{R}_{l m}}{d v} d A \\
& +\frac{a^{2}}{v^{2}} \iint_{A} \frac{l^{2}}{r^{2}} 2 R_{l m} \frac{d R_{l m}}{d v} d A-\frac{2 a^{2}}{v^{3}} \iint_{A} \frac{l^{2}}{r^{2}} R_{l m}^{2} d A
\end{aligned}
$$

where $\dot{R}_{l m}=d R_{l m} / d r$ and $d \dot{R}_{l m} / d v=d / d r\left(d R_{l m} / d v\right)$.

Multiplying Eq. (4) by $d R_{l m} / d v$ and integrating over $A$ yields

$$
\frac{a^{2}}{v^{2}} \iint_{A} \frac{d \dot{R}_{l m}}{d v} \dot{R}_{l m} d A=\iint_{c o r e}\left(1-\frac{\Delta(r)}{\Delta_{0}}\right) R_{l m} \frac{d R_{l m}}{d v} d A+\frac{a^{2}}{v^{2}} \iint_{A} \frac{l^{2}}{r^{2}} \frac{d R_{l m}}{d v} R_{l m} d A
$$

Substituting Eq. (10) into Eq. (9), the normalized waveguide dispersion $v d^{2}(v b) /$ $d v^{2}$ can be expressed as 


$$
v \frac{d^{2}(v B)}{d v^{2}}=2 a^{2} \frac{d}{d v}\left[\frac{1}{v}\left\{\iint_{A} \dot{R}_{l m}^{2} d A+\iint_{A} \frac{l^{2}}{r^{2}} R_{l m}^{2} d A\right\} .\right.
$$

When $\left[\left(\lambda / n_{2}\right)\left(d n_{2} / d \lambda\right)\right] \ll 1$, the waveguide contribution to the dispersion of the group delay $\tau$ per unit length of a fiber is [6]

$$
D_{w}=\frac{d \tau}{d \lambda}=-\frac{n_{2} \Delta_{0}}{c \lambda} v \frac{d^{2}(v B)}{d v^{2}},
$$

where $c$ is the velocity of light in vacuum.

Therefore, the waveguide dispersion $D_{w}$ can be expressed as

$$
D_{w}=\frac{d \tau}{d \lambda}=-\frac{n_{2} \Delta_{0}}{c \lambda} v \frac{d^{2}(v B)}{d v^{2}}=-\frac{n_{2} \Delta_{0}}{c \lambda} 2 a^{2} \frac{d}{d v}\left[\frac{1}{v}\left\{\iint_{A} \dot{R}_{l m}^{2} d A+\iint_{A} \frac{l^{2}}{r^{2}} R_{l m}^{2} d A\right\} .\right.
$$

\section{Simple expression for chromatic dispersion}

\subsection{Waveguide dispersion}

To obtain a simple expression for the waveguide dispersion of the $\mathrm{LP}_{l m}$ mode using Eq. (13), the expression for the electric field of the $\mathrm{LP}_{l m}$ mode is required. Here, we approximated the electric field $R_{l m}(r)$ of a given $\mathrm{LP}_{l m}$ mode in the FMFs as follows [4]:

$$
R_{l m}(r)=\frac{2}{r_{l m}} \sqrt{\frac{(m-1) !}{(l+m-1) !}}\left(\frac{\sqrt{2} r}{r_{l m}}\right)^{(l)} \times L_{m-1}^{(l)}\left(\frac{2 r^{2}}{r_{l m}^{2}}\right) \exp \left(-\frac{r^{2}}{r_{l m}^{2}}\right),
$$

where $r_{l m}$ is the spread of the electric field in the $\mathrm{LP}_{l m}$ mode, which corresponds to the higher-order Gaussian spot size [4]. $L_{m-1}^{(l)}(x)$, which is the associated Laguerre polynomial, can be defined as

$$
L_{m-1}^{(l)}(x)=\sum_{i=0}^{m-1}(-1)^{i} \frac{(l+m-1) !}{i !(m-1-i) !(i+l) !} x^{i} .
$$

It should be noted that Eq. (14) is an exact solution for a fiber with an infinite parabolic profile. It has been reported that the measured electric fields are in good agreement with the electric field approximated by Eq. (14) in several FMFs [7].

The approximated expression for the electric field of each $\mathrm{LP}_{l m}$ mode is expressed in terms of $r_{l m}$ using Eqs. (14) and (15). The following definition for the mode field diameter (MFD) is considered a means to evaluate the spread of the electric field [8]:

$$
2 w_{l m}=2\left[2 \frac{\int_{0}^{\infty} R_{l m}{ }^{2}(r) r d r}{\int_{0}^{\infty}\left(\frac{d R_{l m}}{d r}\right)^{2} r d r}\right]^{1 / 2} .
$$

The relationship between $r_{l m}$ and the MFR $w_{l m}$ can be obtained by substituting the approximated electric field $R_{l m}$ of each mode into the MFD definition in Eq. (16). By substituting Eq. (14) into Eq. (13) and using the relationship between $r_{l m}$ and $w_{l m}$, a simple expression for the waveguide dispersion of each mode as a function

of MFR can be obtained. The simple expressions for the lowest four LP modes are summarized in Table I. 
Table I. Waveguide dispersion expression for each mode in FMFs

\begin{tabular}{c|c}
\hline Mode & Waveguide dispersion expression \\
\hline $\mathrm{LP}_{01}$ & $D_{w}=\frac{\lambda}{2 \pi^{2} n_{1} c w_{01}^{2}}\left(1-\frac{2 \lambda}{w_{01}} \frac{d w_{01}}{d \lambda}\right)$ \\
\hline $\mathrm{LP}_{11}$ & $D_{w}=\frac{\lambda}{\pi^{2} n_{1} c w_{11}^{2}}\left(1-\frac{2 \lambda}{w_{11}} \frac{d w_{01}}{d \lambda}\right)$ \\
\hline $\mathrm{LP}_{02}$ & $D_{w}=\frac{\lambda}{2 \pi^{2} n_{1} c w_{02}^{2}}\left(1-\frac{2 \lambda}{w_{02}} \frac{d w_{01}}{d \lambda}\right)$ \\
\hline $\mathrm{LP}_{21}$ & $D_{w}=\frac{3 \lambda}{2 \pi^{2} n_{1} c w_{21}^{2}}\left(1-\frac{2 \lambda}{w_{21}} \frac{d w_{01}}{d \lambda}\right)$ \\
\hline
\end{tabular}

\subsection{Chromatic dispersion}

The chromatic dispersion $D$ of each mode in the FMFs can be expressed as [9]

$$
\begin{aligned}
D & =-\frac{1}{c \lambda}\left[k \frac{d N_{2}}{d k}+k \frac{d\left(N_{1}-N_{2}\right)}{d k} \frac{d(v b)}{d v}+\left(N_{1}-N_{2}\right) v \frac{d^{2}(v b)}{d v^{2}}\right] \\
& \simeq-\frac{1}{c \lambda}\left[k \frac{d N_{1}}{d k}+\left(N_{1}-N_{2}\right) v \frac{d^{2}(v b)}{d v^{2}}\right]=D_{m}+D_{w} \quad\left(\text { when } \frac{d(v b)}{d v} \simeq 1\right)
\end{aligned}
$$

where $N_{1}$ and $N_{2}$ denote the group indices of the core and cladding, respectively.

In this case, $D$ can be approximately expressed as the sum of the dispersions of the waveguide $\left(D_{w}\right)$ and material $\left(D_{m}\right)$ when $d(v b) / d \lambda$ is approximately 1 .

$D_{m}$ can be defined as

$$
D_{m}=-\frac{\lambda}{c} \frac{d^{2} n}{d \lambda^{2}} .
$$

In particular, the material dispersion is estimated from the Sellmeier formula in which the coefficients correspond to the relative-index difference $\Delta$.

\subsection{Simulations}

Numerical simulations were performed to verify the accuracy of the simple expression in Table I to estimate the chromatic dispersion of each mode in an FMF. A four-mode fiber with $a$ of $12 \mu \mathrm{m}$ and $\Delta$ of $0.32 \%$ was considered, along with a step-index profile.

Figures 1(a)-1(d) show the estimated and calculated chromatic dispersions as a function of the wavelength for the $\mathrm{LP}_{01}, \mathrm{LP}_{11}, \mathrm{LP}_{02}$, and $\mathrm{LP}_{21}$ modes. Furthermore, to evaluate the accuracy of the proposed expression, we estimated the error of the chromatic dispersion for each mode, as shown in Fig. 1. It can be observed that the estimated chromatic dispersions for the $\mathrm{LP}_{01}, \mathrm{LP}_{11}$, and $\mathrm{LP}_{02}$ modes are in good agreement with the calculated values. The estimated accuracy was within $0.8 \mathrm{ps} / \mathrm{km} / \mathrm{nm}$ in the wavelength region between $1.2 \mu \mathrm{m}$ and $1.6 \mu \mathrm{m}$. Although the estimated error for the $\mathrm{LP}_{21}$ mode was determined to be less than $4 \mathrm{ps} / \mathrm{km} / \mathrm{nm}$, it was larger than those of the other modes. This error is probably due to the difference between the actual electric field of the $\mathrm{LP}_{21}$ mode and the electric field approximated using Eqs. (14) and (16). It is known that the MFD values of higher-order modes depend on the definition for obtaining the MFD [7]; therefore, we need to clarify the appropriate definition in the near future to improve the estimation accuracy. Nevertheless, these results indicate that the proposed technique can estimate the 
chromatic dispersion of a high order mode in a four-mode fiber with an accuracy of less than $4 \mathrm{ps} / \mathrm{km} / \mathrm{nm}$.

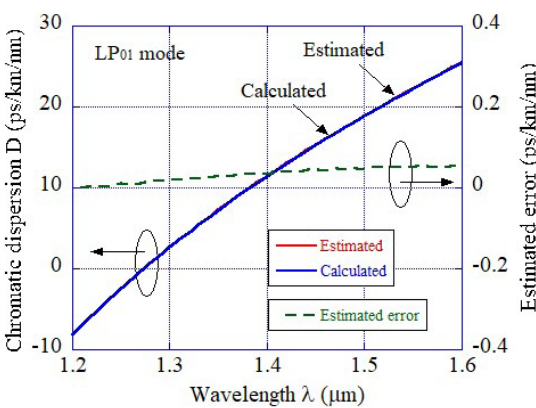

(a) $\mathrm{LP}_{01}$ mode

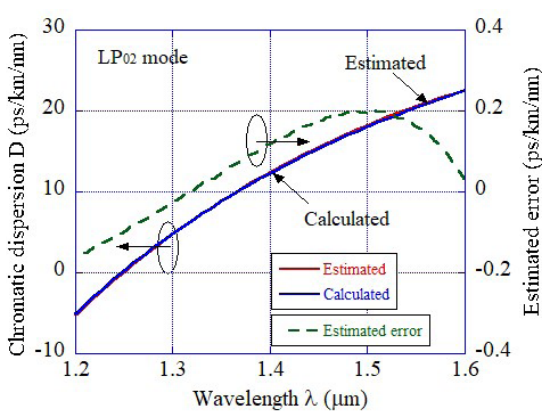

(c) $\mathrm{LP}_{02}$ mode

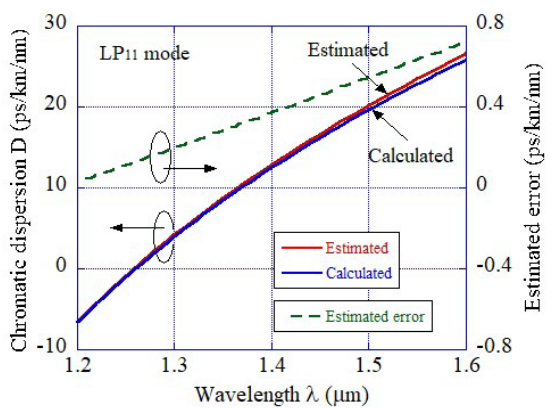

(b) $\mathrm{LP}_{11}$ mode

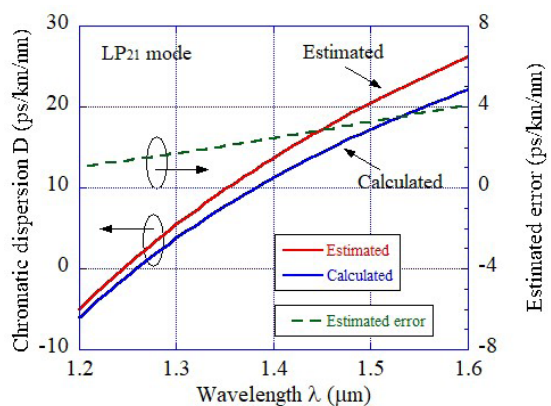

(d) $\mathrm{LP}_{21}$ mode

Fig. 1. Comparison of estimated and calculated chromatic dispersions and the estimated error for each mode in a four-mode fiber

\section{Conclusion}

A general expression for the waveguide dispersion was derived, which was applied to the high-order $\mathrm{LP}_{l m}$ mode in FMFs. Furthermore, a simple expression for chromatic dispersion, which is composed of waveguide and material dispersions, was derived from the general expression. The chromatic dispersions of the $\mathrm{LP}_{01}, \mathrm{LP}_{11}, \mathrm{LP}_{02}$, and $\mathrm{LP}_{21}$ modes in a four-mode fiber were estimated using the proposed expressions. These chromatic dispersions could be successfully estimated with an accuracy of less than $1 \mathrm{ps} / \mathrm{km} / \mathrm{nm}$, except in the case of $\mathrm{LP}_{21}$ mode in which the estimated accuracy was less than $4 \mathrm{ps} / \mathrm{km} / \mathrm{nm}$. Future work will focus on improving the proposed technique.

\section{Acknowledgments}

Part of this research is based on results from "Research and development of innovative optical fiber for practical use" commissioned by the National Institute of Information and Communications Technology.

We thank Hiroyuki Oshida, the Project Manager of NTT Access Network Service Systems Laboratories, for his valuable comments and frequent encouragement. 